\title{
ASYMPTOTIC BEHAVIOUR FOR A NONLOCAL DIFFUSION EQUATION ON A LATTICE
}

\author{
LIVIU I. IGNAT AND JULIO D. ROSSI
}

\begin{abstract}
In this paper we study the asymptotic behaviour as $t \rightarrow \infty$ of solutions to a nonlocal diffusion problem on a lattice, namely, $u_{n}^{\prime}(t)=\sum_{j \in \mathbb{Z}^{d}} J_{n-j} u_{j}(t)-u_{n}(t)$ with $t \geq 0$ and $n \in \mathbb{Z}^{d}$. We assume that $J$ is nonnegative and verifies $\sum_{n \in \mathbb{Z}^{d}} J_{n}=1$. We find that solutions decay to zero as $t \rightarrow \infty$ and prove an optimal decay rate using, as our main tool, the discrete Fourier transform.
\end{abstract}

\section{INTRODUCTION}

In this paper our main concern is the study of the asymptotic behaviour of the following nonlocal equation on a lattice

$$
\begin{cases}u_{n}{ }^{\prime}(t)=(J * u)_{n}(t)-u_{n}(t), & t \geq 0, n \in \mathbb{Z}^{d} \\ u_{n}(0)=\varphi_{n}, & n \in \mathbb{Z}^{d}\end{cases}
$$

where by $(J * u)$ we denote the discrete convolution,

$$
(J * u)_{n}=\sum_{j \in \mathbb{Z}^{d}} J_{n-j} u_{j} .
$$

Trough the paper we assume that the kernel $J$ is nonnegative and satisfies,

$$
\sum_{n \in \mathbb{Z}^{d}} J_{n}=1
$$

Equation (1.1), is called nonlocal diffusion equation. Continuous analogous to (1.1), like $u_{t}(x, t)=J * u(x, t)-u(x, t)$, have been recently widely used to model diffusion processes, see, for example, [2], [3], [5], 6], [8], 9], [10], [16] and [17]. In particular, let us mention that these equations are also used in models of neuronal activity, see [7, [11, [13] and [14]. Also there is a discrete counterpart for nonlocal models, see [1], [3] and references therein. In all these models the asymptotic behaviour of the solution (see [4]) is relevant, both from its pure mathematical and its applied point of view. Concerning (1.1), as stated in [9] (see also [3]), if $u_{i}(t)$ is thought of as the density of a single population at the point $i$ at time $t$, and $J_{i-j}$ is thought of as the probability distribution of jumping from location $i$ to location $j$, then $(J * u)(t)$ is the rate at which individuals are arriving to position $i$ from all other places and $-u_{i}(t)$ is the rate at which they are leaving location $i$ to travel to all other sites. This consideration, in the absence of external or internal sources, leads immediately to the fact that the density $u$ satisfies equation (1.1).

To study the asymptotic beahviour of solutions to (1.1) let us introduce the discrete Laplacian given by

$$
\left(\Delta_{d} u\right)_{n}=\sum_{k=1}^{d}\left(u_{n+e_{k}}-2 u_{n}+u_{n-e_{k}}\right)
$$


where $\left\{e_{k}\right\}_{k=1}^{d}$ is the canonical basis on $\mathbb{R}^{d}$. Note that this is a local diffusion operator.

Our first result says that the asymptotic behaviour as $t \rightarrow \infty$ of solutions to (1.1) is the same as the one for the evolution equation associated to a fractional power of the discrete Laplacian.

Theorem 1.1. Let $u$ be a solution of equation (1.1) with $\varphi \in l^{1}\left(\mathbb{Z}^{d}\right)$. If there exist positive constants $\alpha$ and $A$ such that

$$
\widehat{J}(\xi)=1-A|\xi|^{\alpha}+o\left(|\xi|^{\alpha}\right), \quad \text { as } \xi \rightarrow 0,
$$

then the asymptotic behaviour of $u(t)$ is given by

$$
\lim _{t \rightarrow \infty} t^{d / \alpha}\|u(t)-v(t)\|_{l^{\infty}\left(\mathbb{Z}^{d}\right)}=0
$$

where $v$ is solution of $v^{\prime}=-A\left(-\Delta_{d}\right)^{\alpha / 2} v$ with initial datum $v_{n}(0)=\varphi_{n}, n \in \mathbb{Z}^{d}$.

In view of this result, we analyze the asymptotic profile of the solutions to $v^{\prime}=-A\left(-\Delta_{d}\right)^{\alpha / 2} v$.

Theorem 1.2. Let us consider $\varphi \in l^{1}\left(\mathbb{Z}^{d}\right)$. Then the solution to

$$
\left\{\begin{array}{l}
v^{\prime}(t)=-A\left(-\Delta_{d}\right)^{\alpha / 2} v, \quad t>0 \\
v(0)=\varphi
\end{array}\right.
$$

satisfies

$$
\lim _{t \rightarrow \infty} \sup _{j \in \mathbb{Z}^{d}}\left|t^{d / \alpha} v\left(\left[j t^{1 / \alpha}\right], t\right)-\left(\sum_{n \in \mathbb{Z}^{d}} \varphi_{n}\right) G^{A}(j)\right|=0,
$$

where $G^{A}$ is defined by

$$
G^{A}(x)=\int_{\mathbb{R}^{d}} e^{i x \xi} e^{-A|\xi|^{\alpha}} d \xi
$$

and $[\cdot]$ is the floor function.

\section{Proofs of the Results}

In our analysis, we make use of the semidiscrete Fourier transform (SDFT) (we refer to [12] and [15] for the mains properties of the SDTF). For any $v \in l^{2}\left(\mathbb{Z}^{d}\right)$ we define its SDFT by:

$$
\widehat{v}(\xi)=\sum_{j \in \mathbb{Z}^{d}} e^{-i \xi \cdot j} v_{j}, \quad \xi \in[-\pi, \pi]^{d} .
$$

In view of property (1.2), $\widehat{J}$ belongs to $L^{\infty}([-\pi, \pi])$ and $\widehat{J}(0)=1$.

Proof of Theorem 1.1. Applying the SDFT to the solutions of equation (1.1) we get

$$
\widehat{u}^{\prime}(t, \xi)=\widehat{J}(\xi) \widehat{u}(t, \xi)-\widehat{u}(t, \xi), \quad \xi \in[-\pi, \pi]^{d}, t>0 .
$$

Solving this ODE we find that

$$
\widehat{u}(t, \xi)=e^{t(\widehat{J}(\xi)-1)} \widehat{\varphi}(\xi), \quad \xi \in[-\pi, \pi]^{d}, t>0 .
$$

In the same way, $v$, the solution to $v^{\prime}=-A\left(-\Delta_{d}\right)^{\alpha / 2} v$ satisfies

$$
\widehat{v}(t, \xi)=e^{-A t p^{\alpha}(\xi)} \widehat{\varphi}(\xi), \quad \xi \in[-\pi, \pi]^{d}, t>0,
$$


where

$$
p(\xi)=\left(4 \sum_{k=1}^{d} \sin ^{2}\left(\frac{\xi_{k}}{2}\right)\right)^{1 / 2} .
$$

Using the Fourier representation of $u$ and $v$ given by (2.1) and (2.2) we find that

$$
\begin{aligned}
\|u(t)-v(t)\|_{l^{\infty}\left(\mathbb{Z}^{d}\right)} & \leq \int_{[-\pi, \pi]^{d}}|\widehat{u}(\xi, t)-\widehat{v}(\xi, t)| d \xi \\
& =\int_{[-\pi, \pi]^{d}}\left|\exp (t(\widehat{J}(\xi)-1))-\exp \left(-A t p^{\alpha}(\xi)\right) \| \widehat{\varphi}(\xi)\right| d \xi .
\end{aligned}
$$

By our hypothesis there exists a positive $R<\pi$ such that

$$
|\widehat{J}(\xi)| \leq 1-\frac{|\xi|^{\alpha}}{2},|\xi| \leq R
$$

Once $R$ has been fixed, there exists $\delta>0$ such that

$$
|\widehat{J}(\xi)| \leq 1-\delta \quad \text { for all } \xi \in \Omega_{R}=\left\{\xi \in[-\pi, \pi]^{d},|\xi|>R\right\} .
$$

Hence, it is easy to see that

$$
\begin{gathered}
\int_{\xi \in \Omega_{R}}\left|e^{t(\widehat{J}(\xi)-1)}-e^{-A t p^{\alpha}(\xi)}\|\widehat{\varphi}(\xi) \mid d \xi \leq\| \widehat{\varphi} \|_{L^{\infty}\left([-\pi, \pi]^{d}\right)} \int_{\xi \in \Omega_{R}}\left(e^{t(|\widehat{J}(\xi)|-1)}+e^{-A t p^{\alpha}(\xi)}\right) d \xi\right. \\
\leq\|\widehat{\varphi}\|_{L^{\infty}\left([-\pi, \pi]^{d}\right)} \int_{\xi \in \Omega_{R}}\left(e^{-t \delta}+\exp \left(-A t \inf _{\xi \in \Omega_{R}} p^{\alpha}(\xi)\right) d \xi .\right.
\end{gathered}
$$

Tacking into account that the right hand side in the last inequality is exponentially small, it remains to analyze the term

$$
I(t)=\int_{|\xi| \leq R}|\widehat{u}(\xi, t)-\widehat{v}(\xi, t)| d \xi
$$

Let us choose a function $r(t) \rightarrow 0$ such that $r(t) t^{1 / \alpha} \rightarrow \infty$ as $t \rightarrow \infty$. The remaining term $I(t)$ satisfies:

$$
I(t)=\int_{|\xi| \leq R}\left|e^{t(\widehat{J}(\xi)-1)}-e^{-A t p^{\alpha}(\xi)}\right||\widehat{\varphi}(\xi)| d \xi \leq I_{1}(t)+I_{2}(t)
$$

where

$$
I_{1}(t)=\int_{|\xi| \leq r(t)}\left|e^{t(\widehat{J}(\xi)-1)}-e^{-A t p^{\alpha}(\xi)}\right||\widehat{\varphi}(\xi)| d \xi
$$

and

$$
I_{2}(t)=\int_{r(t) \leq|\xi| \leq R}\left|e^{t(\widehat{J}(\xi)-1)}-e^{-A t p^{\alpha}(\xi)} \| \widehat{\varphi}(\xi)\right| d \xi .
$$

Using that, for some positive constant $c$, the following holds

$$
c|\xi| \leq p(\xi) \leq|\xi| \quad \text { for all } \xi \in[-\pi, \pi]^{d},
$$


the term $I_{2}(t)$ can be estimated as follows:

$$
\begin{aligned}
t^{d / \alpha} I_{2}(t) & \leq t^{d / \alpha}\|\widehat{\varphi}\|_{L^{\infty}\left([-\pi, \pi]^{d}\right)} \int_{r(t) \leq|\xi| \leq R}\left(e^{-A t p^{\alpha}(\xi)}+e^{t(|\widehat{J}(\xi)|-1)}\right) d \xi \\
& \leq t^{d / \alpha}\|\varphi\|_{L^{1}\left(\mathbb{Z}^{d}\right)} \int_{r(t) \leq|\xi| \leq R}\left(e^{-A t p^{\alpha}(\xi)}+e^{-t|\xi|^{\alpha} / 2}\right) d \xi \\
& \leq t^{d / \alpha}\|\varphi\|_{L^{1}\left(\mathbb{Z}^{d}\right)} \int_{r(t) \leq|\xi| \leq R} e^{-B t|\xi|^{\alpha}} d \xi \\
& =\|\varphi\|_{L^{1}\left(\mathbb{Z}^{d}\right)} \int_{t^{1 / \alpha} r(t)|\xi| \leq t^{1 / \alpha}} e^{-B|\xi|^{\alpha}} d \xi \\
& \leq\|\varphi\|_{L^{1}\left(\mathbb{Z}^{d}\right)} t^{d / \alpha} e^{-B t r^{\alpha}(t)} \rightarrow 0 .
\end{aligned}
$$

To estimate $I_{1}(t)$ we first observe that there exists a function $h(\xi)$ with $h(\xi) \rightarrow 0$ as $|\xi| \rightarrow 0$ and such that

$$
\left.|\widehat{J}(\xi)-1-A| \xi\right|^{\alpha}|\leq| \xi \mid h(\xi)
$$

for all $\xi$ in a sufficiently small ball centered at the origin. Thus for all such $\xi$

$$
\left|\widehat{J}(\xi)-1-A p^{\alpha}(\xi)\right| \leq|\xi|^{\alpha} h(\xi)+\left.|| \xi\right|^{\alpha}-\left.p^{\alpha}(\xi)|\lesssim| \xi\right|^{\alpha} h(\xi)+|\xi|^{3 \alpha}
$$

In view of this property we get

$$
\begin{aligned}
I_{1}(t) & \leq t^{d / \alpha}\|\widehat{\varphi}\|_{L^{\infty}\left([-\pi, \pi]^{d}\right)} \int_{|\xi| \leq r(t)} e^{-A t p^{\alpha}(\xi)}\left|e^{t\left(\widehat{J}(\xi)-1-A t p^{\alpha}(\xi)\right)}-1\right| d \xi \\
& \leq t^{d / \alpha}\|\varphi\|_{L^{1}\left(\mathbb{Z}^{d}\right)} \int_{|\xi| \leq r(t)} e^{-A t p^{\alpha}(\xi)} t|\xi|^{\alpha}\left(h(\xi)+|\xi|^{3 \alpha}\right) d \xi \\
& \leq t^{d / \alpha}\|\varphi\|_{L^{1}\left(\mathbb{Z}^{d}\right)} \int_{|\xi| \leq r(t)} e^{-B t|\xi|^{\alpha}}\left(t|\xi|^{\alpha} h(\xi)+t|\xi|^{4 \alpha}\right) d \xi .
\end{aligned}
$$

The last term in the right hand side verifies

$$
t^{d / \alpha} \int_{|\xi| \leq r(t)} e^{-B t|\xi|^{\alpha}} t|\xi|^{4 \alpha} d \xi \leq t^{-3} \int_{|\eta| \leq r(t) t^{1 / \alpha}} e^{-B|\eta|^{\alpha}}|\eta|^{4 \alpha} d \eta \rightarrow 0 .
$$

Hence we have to analyze the first one. In this case, by the same change of variables, we get

$$
t^{d / \alpha} \int_{|\xi| \leq r(t)} e^{-B t|\xi|^{\alpha}} t|\xi|^{\alpha} h(\xi)=\int_{|\eta|<r(t) t^{1 / \alpha}}|\eta|^{\alpha} e^{-B t|\eta|^{\alpha}} h\left(\eta t^{-1 / \alpha}\right) .
$$

Applying Lebesgue convergence theorem we obtain that also this term converges to zero as $t \rightarrow \infty$. This ends the proof.

Now we prove our second result, Theorem 1.2, that describes the asymptotic profile of solutions to $v^{\prime}=-A\left(-\Delta_{d}\right)^{\alpha / 2} v$.

Proof of Theorem 1.2. Using the Fourier representation of $v$ we have

$$
v(j, t)=\int_{[-\pi, \pi]^{d}} e^{-A t p^{\alpha}(\xi)} e^{i j \xi} \widehat{\varphi}(\xi) d \xi, \quad j \in \mathbb{Z}^{d}, t>0 .
$$


Thus

$$
\begin{aligned}
t^{d / \alpha} v\left(\left[j t^{1 / \alpha}\right], t\right) & =t^{d / \alpha} \int_{[-\pi, \pi]^{d}} e^{-A t p^{\alpha}(\xi)} e^{i\left[j t^{1 / \alpha}\right] \xi} \widehat{\varphi}(\xi) d \xi \\
& =\int_{\left[-\pi t^{1 / \alpha}, \pi t^{1 / \alpha}\right]^{d}} e^{-A t p^{\alpha}\left(\xi t^{-1 / \alpha}\right)} \exp \left(i \xi \frac{\left[j t^{1 / \alpha}\right]}{t^{1 / \alpha}}\right) \widehat{\varphi}\left(\xi t^{-1 / \alpha}\right) d \xi
\end{aligned}
$$

and

$$
G^{A}(j)=\int_{\mathbb{R}^{d}} e^{-A|\xi|^{\alpha}} e^{i \xi j} d \xi
$$

Denoting

$$
I(j, t)=t^{d / \alpha} v\left(\left[j t^{1 / \alpha}\right], t\right)-\widehat{\varphi}(0) G^{A}(j)
$$

we obtain

$$
\begin{aligned}
|I(j, t)| \leq & \left|\int_{\left[-\pi t^{1 / \alpha}, \pi t^{1 / \alpha}\right]^{d}} e^{-A t p^{\alpha}\left(\xi t^{-1 / \alpha}\right)} e^{i j \xi}-\widehat{\varphi}(0) \int_{\mathbb{R}^{d}} e^{-A|\xi|^{\alpha}} e^{i \xi j} d \xi\right| \\
& +\int_{\left[-\pi t^{1 / \alpha}, \pi t^{1 / \alpha}\right]^{d}} e^{-A t p^{\alpha}\left(\xi t^{-1 / \alpha}\right)}\left|e^{i j \xi}-e^{i \xi\left[j t^{1 / \alpha}\right] t^{-1 / \alpha}}\right|\left|\widehat{\varphi}\left(\xi t^{-1 / \alpha}\right)\right| d \xi \\
= & I_{1}(j, t)+I_{2}(j, t) .
\end{aligned}
$$

Therefore we have to get bounds for $I_{1}(j, t)$ and $I_{2}(j, t)$.

Step I. Estimates for $I_{2}(t)$. For $I_{2}(t)$ we have the rough estimate

$$
\begin{aligned}
\left|I_{2}(j, t)\right| & \leq\|\widehat{\varphi}\|_{L^{\infty}\left([-\pi, \pi]^{d}\right)} \int_{\left[-\pi t^{1 / \alpha}, \pi t^{1 / \alpha}\right]^{d}} e^{-A t p^{\alpha}\left(\xi t^{-1 / \alpha}\right)}\left|\sin \left(\frac{j t^{1 / \alpha} \xi-\left[j t^{1 / \alpha}\right] \xi}{2 t^{1 / \alpha}}\right)\right| d \xi \\
& \leq\|\varphi\|_{L^{1}\left(\mathbb{Z}^{d}\right)} \int_{\left[-\pi t^{1 / \alpha}, \pi t^{1 / \alpha}\right]^{d}} e^{-A t p^{\alpha}\left(\xi t^{-1 / \alpha}\right)}\left|\frac{j t^{1 / \alpha} \xi-\left[j t^{1 / \alpha}\right] \xi}{2 t^{1 / \alpha}}\right| d \xi \\
& \leq\|\varphi\|_{L^{1}\left(\mathbb{Z}^{d}\right)} \int_{\left[-\pi t^{1 / \alpha}, \pi t^{1 / \alpha}\right]^{d}} e^{-A t p^{\alpha}\left(\xi t^{-1 / \alpha}\right)} \frac{|\xi|}{t^{1 / \alpha}} d \xi \\
& \lesssim t^{-1 / \alpha}\|\varphi\|_{L^{1}\left(\mathbb{Z}^{d}\right)} \int_{\left[-\pi t^{1 / \alpha}, \pi t^{1 / \alpha}\right]^{d}} e^{-c(\alpha)|\xi|^{\alpha}}|\xi| d \xi \rightarrow 0 .
\end{aligned}
$$

Step II. Estimates for $I_{1}(t)$. Observe that $I_{1}$ satisfies:

$$
\begin{aligned}
I_{1}(j, t) \leq & \int_{\left[-\pi t^{1 / \alpha}, \pi t^{1 / \alpha}\right]^{d}}\left|e^{-A t p^{\alpha}\left(\xi t^{-1 / \alpha}\right)}-e^{-A|\xi|^{\alpha}}\right|\left|\widehat{\varphi}\left(\xi t^{-1 / \alpha}\right)\right| d \xi \\
& +\int_{\left[-\pi t^{1 / \alpha}, \pi t^{1 / \alpha}\right]^{d}} e^{-A|\xi|^{\alpha}}\left|\widehat{\varphi}\left(\xi t^{-1 / \alpha}\right)-\widehat{\varphi}(0)\right| d \xi \\
& +|\widehat{\varphi}(0)| \int_{\xi \notin\left[-\pi t^{1 / \alpha}, \pi t^{1 / \alpha}\right]^{d}} e^{-A|\xi|^{\alpha}} d \xi \\
= & I_{3}(t)+I_{4}(t)+I_{5}(t) .
\end{aligned}
$$

In the case of the last integral, easily follows that $|\xi| \gtrsim t^{1 / \alpha}$. Thus

$$
I_{5}(t) \lesssim \int_{|\xi| \gtrsim t^{1 / \alpha}} e^{-A|\xi|^{\alpha}} d \xi \rightarrow 0 .
$$


For $I_{4}$ we have the following estimate:

$$
I_{4}(t)=\int_{\mathbb{R}^{d}} e^{-A|\xi|^{\alpha}}\left|\widehat{\varphi}\left(\xi t^{-1 / \alpha}\right)-\widehat{\varphi}(0)\right| \chi_{\left[-\pi t^{1 / \alpha}, \pi t^{1 / \alpha}\right]^{d}} d \xi
$$

and the Lebesgue dominated convergence theorem guarantees that $I_{4}(t) \rightarrow 0$ as $t \rightarrow \infty$.

Using that $p(\xi)$ satisfies $c|\xi| \leq p(\xi) \leq|\xi|$ for some positive $c$ and the mean value theorem we get:

$$
\begin{aligned}
I_{3}(t) & \leq\left.\|\widehat{\varphi}\|_{L^{\infty}\left([-\pi, \pi]^{d}\right)} \int_{\left[-\pi t^{1 / \alpha}, \pi t^{1 / \alpha}\right]^{d}} e^{-A t p^{\alpha}\left(\xi t^{-1 / \alpha}\right)}\left|t p^{\alpha}\left(\xi t^{-1 / \alpha}\right)-\right| \xi\right|^{\alpha} \mid d \xi \\
& \left.\lesssim\|\varphi\|_{L^{1}\left(\mathbb{Z}^{d}\right)} \int_{\left[-\pi t^{1 / \alpha}, \pi t^{1 / \alpha}\right]^{d}} e^{-c|\xi|^{\alpha}}\left|t p^{\alpha}\left(\xi t^{-1 / \alpha}\right)-\right| \xi\right|^{\alpha} \mid d \xi .
\end{aligned}
$$

Applying again the dominated convergence theorem we obtain that $I_{3}(t) \rightarrow 0$ as $t \rightarrow \infty$.

The proof is now complete.

Acknowledgements. L. I. Ignat partially supported by the grants MTM2005-00714 and PROFIT CIT-370200-2005-10 of the Spanish MEC, SIMUMAT of CAM and CEEX-M3-C312677 of the Romanian MEC.

J. D. Rossi partially supported by SIMUMAT (Spain), UBA X066, CONICET and ANPCyT PICT 05009 (Argentina).

\section{REFERENCES}

[1] P. Bates and A. Chmaj. A discrete convolution model for phase transitions. Arch. Rat. Mech. Anal., 150, 281-305, (1999).

[2] P. Bates, P. Fife, X. Ren and X. Wang. Travelling waves in a convolution model for phase transitions. Arch. Rat. Mech. Anal., 138, 105-136, (1997).

[3] C. Carrillo and P. Fife. Spatial effects in discrete generation population models. J. Math. Biol., 50(2), 161-188, (2005).

[4] E. Chasseigne, M. Chaves and J. D. Rossi. Asymptotic behavior for nonlocal diffusion equations. J. Math. Pures Appl., 86, 271-291, (2006).

[5] X Chen. Existence, uniqueness and asymptotic stability of travelling waves in nonlocal evolution equations. Adv. Differential Equations, 2, 125-160, (1997).

[6] C. Cortazar, M. Elgueta and J. D. Rossi. A non-local diffusion equation whose solutions develop a free boundary. Annales Henri Poincaré, 6(2), 269-281, (2005).

[7] R. Curtu and B. Ermentrout. Pattern formation in a network of excitatory and inhibitory cells with adaptation. SIAM J. Appl. Dyn. Syst. 3(3), 191-231, (2004).

[8] F. Da Lio, N. Forcadel and R. Monneau. Convergence of a non-local eikonal equation to anisotropic mean curvature motion. Application to dislocations dynamics. to appear in J. Eur. Math. Soc.

[9] P. Fife. Some nonclassical trends in parabolic and parabolic-like evolutions. Trends in nonlinear analysis, 153-191, Springer, Berlin, 2003.

[10] P. Fife and X. Wang. A convolution model for interfacial motion: the generation and propagation of internal layers in higher space dimensions. Adv. Differential Equations, 3(1), 85-110, (1998).

[11] S. E. Folias and P. C. Bressloff. Breathers in Two-Dimensional Neural Media. Phys. Rev. Letters, 95, 208107, 1-4, (2005).

[12] T. W. Körner. Fourier analysis. Cambridge University Press, Cambridge, 1988.

[13] D. J. Pinto and B. G. Ermentrout. Spatially structured activity in synaptically coupled neuronal networks. I. Traveling fronts and pulses. SIAM J. Appl. Math. 62(1), 206-225, (2001).

[14] D. J. Pinto and B. G. Ermentrout. Spatially structured activity in synaptically coupled neuronal networks. II. Lateral inhibition and standing pulses. SIAM J. Appl. Math. 62(1), 226-243, (2001).

[15] L.N. Trefethen, Spectral methods in MATLAB, Software, Environments and Tools, Society for Industrial and Applied Mathematics, 2000. 
[16] X. Wang. Metaestability and stability of patterns in a convolution model for phase transitions. J. Differential Equations, 183, 434-461, (2002).

[17] L. Zhang. Existence, uniqueness and exponential stability of traveling wave solutions of some integral differential equations arising from neuronal networks. J. Differential Equations, 197(1), 162-196, (2004).

L. I. IGNAT

Departamento de Matemáticas,

U. Autónoma de Madrid,

28049 MADRID, SPAIN

AND

Institute of Mathematics of the Romanian ACAdemy,

P.O.Box 1-764, RO-014700 Bucharest, Romania.

E-mail address: liviu.ignat@uam.es

Web page: http://www.uam.es/liviu.ignat

J. D. Rossi

Depto. Matemática, FCEyN UBA (1428)

Buenos Aires, Argentina.

E-mail address: jrossi@dm.uba.ar

Web page: http://mate.dm.uba.ar/ jrossi 